\title{
Ceftriaxone-resistant Salmonella enterica serotype Typhi in a pregnant traveller returning from Karachi, Pakistan to Denmark, 2019
}

Anne Line Engsbro ${ }^{1}$, Hans Søe Riis Jespersen ${ }^{2}$, Maria Ingeborg Goldschmidt ${ }^{3}$, Sarah Mollerup ${ }^{1}$, Peder Worning ${ }^{1}$, Martin Schou Pedersen $^{1}$, Henrik Westh ${ }^{1,4}$, Uffe Vest Schneider ${ }^{1}$

1. Department of Clinical Microbiology, Copenhagen University Hospital Hvidovre, Hvidovre, Denmark

2. Gastrounit, Medical division, Copenhagen University Hospital Hvidovre, Hvidovre, Denmark

3. Department of Infectious Diseases, Copenhagen University Hospital Hvidovre, Hvidovre, Denmark

4. University of Copenhagen, Copenhagen, Denmark

Correspondence: Anne Line Engsbro (Anne.Line.Engsbro@regionh.dk)

Citation style for this article:

Engsbro Anne Line, Riis Jespersen Hans Søe, Goldschmidt Maria Ingeborg, Mollerup Sarah, Worning Peder, Pedersen Martin Schou, Westh Henrik, Schneider Uffe Vest. Ceftriaxone-resistant Salmonella enterica serotype Typhi in a pregnant traveller returning from Karachi, Pakistan to Denmark, 2019. Euro Surveill. 2019;24(21): pii=1900289. https://doi.org/10.2807/1560-7917.ES.2019.24.21.1900289

We describe a ceftriaxone-resistant Salmonella Typhi bacteraemia in a pregnant woman returning from a family visit in Pakistan. Whole genome sequencing confirmed similarity to a Pakistani outbreak clone. Pregnancy and unawareness of this outbreak delayed appropriate antibiotic therapy. Concurrently, we detected faecal carriage of a carbapenemase-producing Escherichia coli. Awareness of the ongoing outbreak should affect empiric treatment of typhoid fever and hygiene precautions in travellers returning from Pakistan. Meropenem may be warranted in severe cases.

In Denmark, typhoid fever is a rare infection in returning travellers with fewer than 100 cases in our laboratory during the past 20 years. On a global level, the World Health Organization (WHO) estimates between 11 and 22 million cases with 128,000 to 161,000 deaths annually [1]. Since November 2016, an outbreak of Salmonella enterica serotype Typhi resistant to ampicillin, trimethoprim/sulfamethoxazole, chloramphenicol, fluoroquinolones and ceftriaxone has been ongoing in the Sindh Province in Pakistan, especially in Karachi and Hyderabad [2]. We here describe one imported case of ceftriaxone-resistant S. Typhi bacteraemia related to travel to Karachi, Pakistan and detected in Denmark in April 2019.

\section{Case description}

An otherwise healthy 15-week-pregnant woman in her 3os experienced fever, diarrhoea and abdominal pains while visiting relatives in Karachi, Pakistan during March and April 2019. In Pakistan, she had been treated with oral cefixime for 1 week. Upon return to Denmark, 2 weeks after initial symptoms, she presented to the emergency department but was not admitted as she was afebrile with normal pulse and blood pressure. No blood tests were done. Two days later, her family doctor admitted her to the Copenhagen University Hospital Hvidovre with fever and abdominal pain. The Figure shows the sequence of antibiotic treatment starting from admission, with corresponding daily level of C-reactive protein (CRP; normal $<10 \mathrm{mg} / \mathrm{L}$ ) and body temperature.

The day after admission (Day 2), an in-house PCR on a rectal swab was positive for Salmonella spp. Based on this finding and because fever and tachycardia continued, treatment with intravenous (iv) ceftriaxone 2 g once daily was started. On Day 4, Salmonella spp. was detected by direct matrix-assisted laser desorption/ionization (MALDI Sepsityper, Bruker, Billerica, United States (US)) in blood cultures drawn on Day 2 (Bactec, BD Diagnostics, New Jersey, US). On Day 5, S. Typhi was identified by agglutination test (Salmonella Antisera, SSI Diagnostica, Hilleroed, Denmark). Based on the results of antibiotic susceptibility testing (AST), reported below, treatment was changed to iv mecillinam ( $1 \mathrm{~g}$ three times/day), a decision also taken in consideration of the pregnancy since penicillins are generally considered safe during pregnancy. The patient deteriorated on this treatment and on Day 8, treatment was changed to azithromycin $(500 \mathrm{mg} / \mathrm{day}$ iv). Because of continuing fever and further increase in CRP, meropenem ( $1 \mathrm{~g}$ three times/day) was added on Day 9. Subsequently, clinical improvement with defervescence and decreasing CRP was seen.

After finding the ceftriaxone-resistant S. Typhi, carriage of other multiresistant microorganisms was suspected and the patient was screened for faecal carriage of carbapenemase-producing microorganisms. 
Body temperature, C-reactive protein level, antibiotic treatment and microbiological diagnostics during and after admission in a pregnant traveller returning with typhoid fever from Pakistan to Denmark, April-May 2019

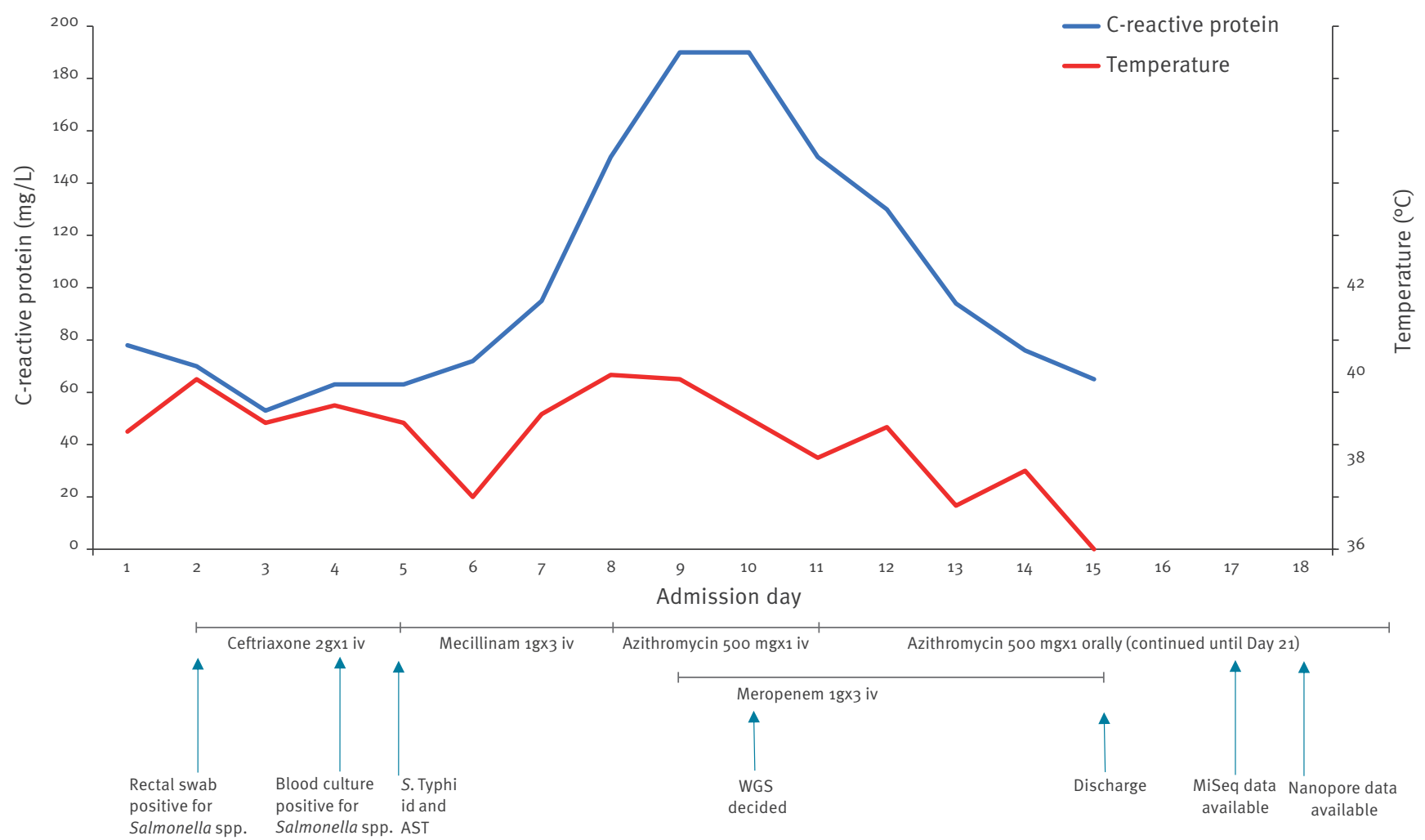

AST: antibiotic susceptibility testing; id: identification; iv: intravenous; WGS: whole genome sequencing.

Antibiotic treatment and microbiological findings are listed underneath the graph.

After the detection of an OXA-48 carbapenemase-producing Escherichia coli, the patient was isolated to prevent spread.

The patient was discharged on Day 15 with oral azithromycin. The fetus remained healthy.

\section{Characterisation of the Salmonella Typhi isolate}

The AST results, determined by disc diffusion and E-test methods and interpreted according to the European Committee on Antimicrobial Susceptibility Testing (EUCAST) breakpoints (version 9.0) [3], are presented in the Table. They confirmed the ceftriaxone-resistant phenotype of the isolate.

By MiSeq (Illumina) and MinION (Oxford Nanopore Technologies, Oxford, United Kingdom (UK)) sequencing, we assembled and closed the genome (BioProject Accession PRJNA543969) and compared it to the $\mathrm{H}_{5} 8$ ceftriaxone-resistant S. Typhi reported from Pakistan (22420_1_10_Pak600006_2016, GenBank accession LT882486) [4]. Our isolate had a chromosome of $4,813,117 \mathrm{bp}$ and a plasmid of $84,498 \mathrm{bp}$ length. The chromosome carried resistance genes corresponding to the $\mathrm{H}_{58} 8$-associated composite transposon antimicrobial resistance (AMR) cassette conferring resistance to the older first-line treatments ampicillin, sulfamethoxazole/trimethoprim and chloramphenicol (multidrugresistant phenotype) and reduced susceptibility of fluoroquinolones (Table). The plasmid was identical to the plasmid identified in the outbreak clone; an IncY plasmid carrying a CTX-M-15 gene and a qnrS1 gene conferring resistance to $\beta$-lactams including ceftriaxone and to ciprofloxacin, respectively. Resistance genes for aminoglycosides other than gentamicin were carried on both chromosome and plasmid. A single nucleotide polymorphism (SNP) analysis showed 0 SNPs difference to the Pakistan outbreak clone. We used the Northern Arizona SNP pipeline (NASP) [5] with Salmonella enterica serovar Typhi $\mathrm{CT}_{18}$ [6] as the reference genome for the SNP analysis.

In a faecal swab, we identified carriage of an OXA48 carbapenemase-producing $E$. coli using the GeneXpert Xpert Carba-R (Cepheid, Sunnyvale, US) and an overnight broth with added meropenem in a concentration of $0.25 \mathrm{mg} / \mathrm{L}$ with subsequent plating on a chromID CARBA-SMART agar (bioMérieux, Marcy l’Etoile, France).

\section{Discussion}

An outbreak of extended-spectrum $\beta$-lactamase producing (ESBL), ciprofloxacin-resistant $S$. Typhi is ongoing 
Antibiotic resistance pattern in a ceftriaxone-resistant Salmonella enterica serotype Typhi detected in Denmark, 2019

\begin{tabular}{|c|c|c|c|c|}
\hline \multirow[b]{2}{*}{ Antibiotic } & \multicolumn{3}{|c|}{ Phenotype } & \multirow{2}{*}{$\begin{array}{c}\text { Genotype } \\
\text { Resistance gene or point mutation } \\
\text { detected (location on the chromosome } \\
\text { (c) and/or the plasmid (p)) }\end{array}$} \\
\hline & $\begin{array}{l}\text { Zone diameter } \\
\quad(\mathrm{mm})\end{array}$ & $\mathrm{MIC}(\mathrm{mg} / \mathrm{L})$ & Interpretation $\mathrm{S}-\mathrm{I}-\mathrm{R}^{\mathrm{a}}$ & \\
\hline Mecillinam & 26 & NT & $\mathrm{s}$ & None \\
\hline Ampicillin & 6 & NT & $R$ & blaTEM-1B $(c+p)$ \\
\hline Amoxicillin/clavulanic acid & 19 & NT & $\mathrm{s}$ & None \\
\hline Piperacillin/tazobactam & 22 & NT & S & None \\
\hline Cefpodoxime & 6 & NT & $\mathrm{R}$ & blaCTX-M-15 (p) \\
\hline Ceftriaxone & NT & $>32$ & $\mathrm{R}$ & blaCTX-M-15 (p) \\
\hline Ceftolozane/tazobactam & NT & 1.0 & $S$ & None \\
\hline Ceftazidime/avibactam & NT & 0.25 & S & None \\
\hline Meropenem & 32 & NT & $S$ & None \\
\hline Gentamicin & 24 & NT & S & None \\
\hline Aminoglycosides other than gentamicin & NT & NT & NT & $\begin{array}{c}\operatorname{aph}[3]-1 b^{b}(c+p) \\
\text { aph[6 }(c)-1 d^{c}(c+p) \text { aac[6]- }\end{array}$ \\
\hline Ciprofloxacin & 20 & NT & $\mathrm{R}$ & gyrA S83F (c) qnrS1 (p) \\
\hline Azithromycin & NT & 2 & $\mathrm{~S}$ & None \\
\hline Trimetoprim/sulfamethoxazole & 6 & NT & $\mathrm{R}$ & $\operatorname{sul}_{1}(c) \operatorname{sul}_{2}(c+p)$ dfrA7 $_{7}(c)$ \\
\hline Aztreonam & NT & $>256$ & $\mathrm{R}$ & blaCTX-M-15 (p) \\
\hline Tigecycline & NT & 0.25 & $\mathrm{~s}$ & None \\
\hline Colistin & NT & 0.125 & $\mathrm{~S}$ & None \\
\hline Chloramphenicol & NT & NT & NT & catA1 (c) \\
\hline
\end{tabular}

c: chromosome; I: intermediate sensitivity; MIC: minimum inhibitory concentration; NT: not tested; p: plasmid; R: resistant; S: sensitive.

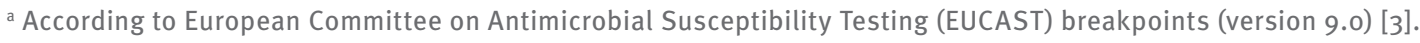

${ }^{b}$ Also called strA.

${ }^{c}$ Also called strB.

in Pakistan. Almost 7,000 cases have been reported in the outbreak [7], and one third of all S. Typhi isolates from blood cultures in Karachi are currently ceftriaxone-resistant [8]. Only one case of ceftriaxone-resistant $S$. Typhi related to travel to Pakistan has previously been described in the UK [4] and recently five cases in children visiting relatives in Pakistan were reported from the US [9]. Since quinolone resistance increased, ceftriaxone has been the recommended treatment of suspected typhoid fever in the returning traveller [10]. The occurrence of travel-related cases of ceftriaxoneresistant typhoid fever in Europe emphasises the need to reconsider our empirical choice of antibiotics for suspected typhoid fever for patients returning from Pakistan.

Our patient deteriorated on mecillinam treatment although the isolate was susceptible to this drug.
Poor clinical response despite in vitro susceptibility has previously been described for mecillinam [11-13]. Azithromycin with the addition of meropenem cured our patient. The effect of azithromycin on S. Typhi is well documented and comparable to quinolones [14], and azithromycin can be used in pregnancy if necessary. Addition of meropenem rather than piperacillin/ tazobactam was chosen because carbapenems are superior in other ESBL-producing Enterobacterales[15]. The carriage of a carbapenemase-producing $E$. coli was not considered when deciding treatment strategy. Our strategy was informed by the local strategy in Pakistan, where ceftriaxone-resistant $S$. Typhi is treated with azithromycin and carbapenems [2]. Our isolate was in vitro sensitive to the newer drugs ceftolozane/tazobactam and ceftazidime/avibactam, which may represent alternative treatment options if carbapenem resistance dissipates to S.Typhi. In general, there was consistency 
between AST and resistance genes identified in the isolate. For chloramphenicol and aminoglycosides, AST was not performed because these tests are not available at our laboratory.

The only case-control study on typhoid fever in pregnancy is from Pakistan and found no increase in negative pregnancy outcomes [16]. In pregnant women, the mother should be the main focus of treatment and choice of antibiotic should follow the above recommendations. Since fluoroquinolones are contraindicated in the second and third trimester of pregnancy, azithromycin is currently the only available oral treatment for carriers of the ceftriaxone-resistant S. Typhi during pregnancy. Third- and newer generation cephalosporins and meropenem are discouraged in pregnancy because of lack of data. However, in our case, clinical improvement coincided with the addition of meropenem to the azithromycin treatment. The fetus was followed with ultrasound scans and was found to be healthy.

The plasmid carried by the ceftriaxone-resistant S. Typhi has previously been described in $E$. coli from four different continents [4]. The clustering of cases around sewage lines in Hyderabad, Pakistan, suggests that the outbreak can be attributed to drinking water contaminated by sewage [2]. Faecal carriage of resistance genes is prevalent in travellers from South Asia as illustrated by identification of resistance genes in toilet waste from airplanes [17]. Finding a multidrug-resistant $S$. Typhi in a returning traveller indicates exposure to faecally contaminated water or foods, and the clinician must suspect the presence of other multi-resistant gastrointestinal bacteria; as was the case in our patient. Screening for multi-resistant Enterobacterales and hygiene precautions should therefore be considered in hospitalised returning travellers.

\section{Conclusion}

Ceftriaxone-resistant $S$. Typhi is here described in an imported European case. Ceftriaxone resistance needs to be taken into consideration when choosing empirical treatment for patients returning from Pakistan with suspected typhoid fever. Azithromycin and meropenem had clinical effect, while the patient deteriorated on mecillinam. Isolating a multidrug-resistant $S$. Typhi should raise the concern of carriage of other multiresistant microorganisms transmitted by the faecaloral route.

\section{Ethical statement}

The patient gave written informed consent to publication.

\section{Conflict of interest}

None declared.
Authors' contributions

Anne Line Engsbro wrote the initial draft. Hans Søe Riis Jespersen and Maria Ingeborg Goldschmidt had the clinical contact with the patient. Maria Ingeborg Goldschmidt obtained informed consent from the patient. Anne Line Engsbro, Henrik Westh and Uffe Vest Schneider were responsible for the microbiological diagnostics and clinical microbiology counselling on the patient. Martin Schou Pedersen performed the sequencing of the organism. Sarah Mollerup, Peder Worning and Martin Schou Pedersen were responsible for the bioinformatics on the sequenced isolate. All authors revised the manuscript and approved the final draft.

\section{References}

1. World Health Organization (WHO). Typhoid. Geneva: WHO. [Accessed: 6 May 2019]. Available from: https://www.who.int/ immunization/diseases/typhoid/en/

2. Qamar FN, Yousafzai MT, Khalid M, Kazi AM, Lohana H, Karim $\mathrm{S}$, et al. Outbreak investigation of ceftriaxone-resistant Salmonella enterica serotype Typhi and its risk factors among the general population in Hyderabad, Pakistan: a matched case-control study. Lancet Infect Dis. 2018;18(12):136876. https://doi.org/10.1016/S1473-3099(18)30483-3 PMID: 30507460

3. European Committee on Antimicrobial Susceptibility Testing (EUCAST). Clinical Breakpoints - bacteria (version 9.0). Basel: European Society of Clinical Microbiology and Infectious Diseases. [Accessed: 21 May 2019]. Available from: http:// www.eucast.org/clinical_breakpoints/

4. Klemm EJ, Shakoor S, Page AJ, Qamar FN, Judge K, Saeed DK, et al. Emergence of an extensively drug-resistant Salmonella enterica serovar Typhi clone harboring a promiscuous plasmid encoding resistance to fluoroquinolones and third-generation cephalosporins. MBio. 2018;9(1):e00105-18. https://doi. org/10.1128/mBio.00105-18 PMID: 29463654

5. Sahl JW, Lemmer D, Travis J, Schupp JM, Gillece JD, Aziz M, et al. NASP: an accurate, rapid method for the identification of SNPs in WGS datasets that supports flexible input and output formats. Microb Genom. 2016;2(8):e000074. https://doi. org/10.1099/mgen.0.000074 PMID: 28348869

6. Parkhill J, Dougan G, James KD, Thomson NR, Pickard D, Wain J, et al. Complete genome sequence of a multiple drug resistant Salmonella enterica serovar Typhi CT18. Nature. 2001;413(6858):848-52. https://doi.org/10.1038/35101607 PMID: 11677608

7. The World Health Organization Regional Office for the Eastern Mediterranean (WHO Eastern Mediterranean Region). Current outbreaks in the WHO Eastern Mediterranean Region. Cairo: WHO Eastern Mediterranean Region. [Accessed: 6 May 2019]. Available from: http://www.emro.who.int/pandemic-epidemic diseases/outbreaks/index.html

8. Cohen J. 'Frightening' typhoid fever outbreak spreads in Pakistan. Science. 2018;361(6399):214. https://doi. org/10.1126/science.361.6399.214 PMID: 30026207

9. Chatham-Stephens K, Medalla F, Hughes M, Appiah GD, Aubert RD, Caidi H, et al. Emergence of extensively drug resistant Salmonella Typhi infections among travelers to or from Pakistan - United States, 2016-2018. MMWR Morb Mortal Wkly Rep. 2019;68(1):11-3. https://doi.org/10.15585/mmwr. mm6801a3 PMID: 30629573

10. Thwaites GE, Day NP. Approach to fever in the returning traveler. N Engl J Med. 2017;376(6):548-60. https://doi. org/10.1056/NEJMra1508435 PMID: 28177860

11. Fallon RJ, Mandal BK, Mayon-White RT, Scott AC. Assessment of antimicrobial treatment of acute typhoid and paratyphoid fevers in Britain and The Netherlands 1971-1980. J Infect. 1988;16(2):129-34. https://doi.org/10.1016/S01634453(88)93872-8 PMID: 3127475

12. Jones DA, Kudlac H, Edwards IR. Pivmecillinam and relapse of typhoid fever. J Infect Dis. 1982;145(5):773. https://doi. org/10.1093/infdis/145.2.773 PMID: 6281342

13. Mandal BK, Ironside AG, Brennand J. Mecillinam in enteric fever. BMJ. 1979;1(6163):586-7. https://doi.org/10.1136/ bmj.1.6163.586 PMID: 218670

14. Effa EE, Lassi ZS, Critchley JA, Garner P, Sinclair D, Olliaro PL, et al. Fluoroquinolones for treating typhoid and paratyphoid fever (enteric fever). Cochrane Database Syst Rev. 2011; (10):CDoo4530. https://doi.org/10.1002/14651858.CDoo4530. pub4 PMID: 21975746

15. Harris PNA, Tambyah PA, Lye DC, Mo Y, Lee TH, Yilmaz M, et al. Effect of piperacillin-tazobactam vs meropenem on 30-day 
mortality for patients with E coli or Klebsiella pneumoniae

bloodstream infection and ceftriaxone resistance: a

randomized clinical trial. JAMA. 2018;320(10):984-94. https://

doi.org/10.1001/jama.2018.12163 PMID: 30208454

16. Sulaiman K, Sarwari AR. Culture-confirmed typhoid fever and pregnancy. Int J Infect Dis. 2007;11(4):337-41. https://doi. org/10.1016/j.ijid.2006.09.007 PMID: 17321180

17. Nordahl Petersen T, Rasmussen S, Hasman H, Carøe C, Bælum J, Schultz AC, et al. Meta-genomic analysis of toilet waste from long distance flights; a step towards global surveillance of infectious diseases and antimicrobial resistance. Sci Rep. 2015;5(1):11444. https://doi.org/10.1038/srep11444 PMID: 26161690

\section{License, supplementary material and copyright}

This is an open-access article distributed under the terms of the Creative Commons Attribution (CC BY 4.0) Licence. You may share and adapt the material, but must give appropriate credit to the source, provide a link to the licence and indicate if changes were made.

Any supplementary material referenced in the article can be found in the online version.

This article is copyright of the authors or their affiliated institutions, 2019. 